J. Clin. Chem. Clin. Biochem.

Vol. 26, 1988, pp. 223-227

(C) 1988 Walter de Gruyter \& Co.

Berlin - New York

\title{
Semiquantitativer Cystein-Schnelltest Möglichkeit zur Verlaufskontrolle der Ascorbinsäuretherapie bei Cystinurie und Cystinlithiasis
}

\author{
Von W. Berg und $O$. Kilian \\ Klinik und Poliklinik für Urologie am Bereich Medizin der Friedrich-Schiller-Universität Jena, Jena, DDR
}

(Eingegangen am 19. Februar/7. September 1987//3. Februar 1988)

\begin{abstract}
Zusammenfassung: In der vorliegenden Arbeit wird eine Methode zur semiquantitativen Cysteinbestimmung im Urin beschrieben, die auf der Bildung eines rotgefärbten Cystein/Nitroprussid-Komplexsalzes beruht. Das Verfahren ist als Schnelltest zur Verlaufskontrolle der Ascorbinsäuretherapie bei Cystinurikern und Cystinsteinpatienten geeignet. Es garantiert eine akzeptable Reproduzierbarkeit der Werte und ist in jedem klinischchemischen Laboratorium einfach durchführbar. Mit dem beschriebenen $\mathrm{K}_{2} \mathrm{CO}_{3} / \mathrm{Nitroprussid-Test} \mathrm{und} \mathrm{einer}$

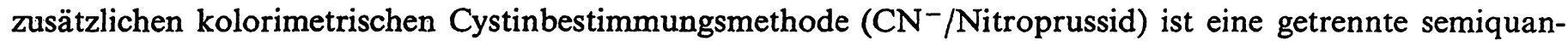
titative Differenzierung von Cystein und Cystin in frischen (!) Urinen möglich.
\end{abstract}

Semiquantitative rapid test for cysteine - A possible means of monitoring ascorbic acid therapy in cystinuria and cystine lithiasis

Summary: A semiquantitative method is presented for the determination of cysteine in urine, based on the formation of a red cysteine/nitroprusside salt. The method is suitable as a rapid test for checking the progress of ascorbic acid therapy of cystinuria and cystine urolithiasis. It guarantees acceptable reproducibility of values and can be easily carried out in any clinical chemical laboratory. With the $\mathrm{K}_{2} \mathrm{CO}_{3}$ /nitroprusside test described and an additional colorimetric determination of cystine ( $\mathrm{CN}^{-} /$nitroprusside), a separate semiquantitative differentiation of cysteine and cystine in fresh (!) urine is possible.

\section{Einführung}

Die Cystinurie gehört zur Gruppe der angeborenen Stoffwechselstörungen (1-3). Dieser Defekt kann sich in einer Cystinsteingenese maǹifestieren. Die relative Häufigkeit der Cystinlithiasis beim Menschen beträgt $0,2-1 \%(2,4,5)$. Zur therapeutischen Anwendung kommen noch immer in der Mehrzahl Sulfhydrylpräparate wie $D$-Penicillamin $\left(\beta, \beta^{\prime}\right.$-Dimethylcystein); Thiola ( $\alpha$-Mercaptopropionylglycin) und Acetylcystein (2, 6-9). Allerdings mehren sich in letzter Zeit auch Hinweise über deren toxische Nebenwirkungen (4, 7, 10-12). Andere therapeutische Maßnahmen beruhen auf der Löslichkeitssteigerung des Cystins durch Alkalisierung des Urins mit $\mathrm{Na}$ triumhydrogencarbonat und/oder Citratgemischen (2, $6,10,13)$. Asper \& Schmucki $(4,14)$ haben eine Therapie mit Ascorbinsäure vorgeschlagen, für die in zahlreichen Studien ein günstiger Effekt auf die Senkung der Rezidivquote von Cystinsteinen ausgewiesen werden konnte (15-18). Das Prinzip dieser recht schonenden Therapie mit hochdosierten Gaben von $5-10 \mathrm{~g} / \mathrm{d}$ beruht auf der Reduktion von Cystin durch Ascorbinsäure zu Cystein, wodurch das molare Cystein-Cystin-Verhältnis im Urin deutlich zugunsten des gut wasserlöslichen Cysteins angehoben werden soll (4). 
In der Literatur ist eine ganze Reihe allerdings $z$. T. apparativ und methodisch aufwendiger Analyseverfahren zur getrennten Bestimmung von Cystin und Cystein zu finden $(4,14,19-24)$. Die nachfolgend beschriebene Schnelltestmethode bietet auf einfache Weise eine laboratoriumsdiagnostisch relevante Möglichkeit zur differenzierten Erfassung von Cystein und Cystin im Urin für klinisch-chemische Verlaufskontrollen bei Ascorbinsäuretherapie.

\section{Material und Methoden}

Cystein-Schnelltest

\section{Reaktionsprinzip und Durchführung}

Die Methode beruht auf dem Prinzip der Brand'schen Reaktion (25), wobei Cystein gemäß Gleichung 1 mit Natrium-pentacyanonitrosylferrat(II) (Natriumnitroprussid) zu einem kirschroten Farbkomplex reagiert.

$$
\begin{aligned}
& \mathrm{R}-\mathrm{S}^{-}+\left[\mathrm{Fe}(\mathrm{CN})_{5} \mathrm{NO}\right]^{2-} \stackrel{\left(\mathrm{K}_{2} \mathrm{CO}_{3}\right)}{\longrightarrow}\left[\mathrm{Fe}(\mathrm{CN})_{5} \mathrm{NOSR}\right]^{3-} \\
& \mathrm{R}=-\mathrm{CH}_{2}-\mathrm{CH}\left(\mathrm{NH}_{2}\right)-\mathrm{COOH}
\end{aligned}
$$

Die Reagenzzusammensetzung wurde einem Cystin-Tüpfeltest nach Maaser et al. (26) entlehnt (s. Tab. 1). Der Zusatz von $\mathrm{K}_{2} \mathrm{CO}_{3}$ garantiert einen für die Farbreaktion ausreichend hohen $\mathrm{pH}$-Wert. Die Cysteinbestimmung erfolgt im Reagenzglas durch Zugabe von $1 \mathrm{ml}$ frischer Urinprobe zur Reagenzmischung. In Abhängigkeit vom Cysteingehalt des Urins tritt in der Reaktionsmischung sofort eine rosa bis tiefkirschrote Färbung auf (vorsichtig schütteln). Gelbliche Färbung der Reagenz/ProbeMischung verweist auf negative Reaktion.

Tab. 1. Such- und Schnelltest für Cystein im Urin - Methodische Details und Kennziffern (vgl. l.c. (26))

\begin{tabular}{ll}
\hline & Pulverreagenz \\
\hline Zusammensetzung & $\mathrm{Na}_{2}\left[\mathrm{Fe}(\mathrm{CN})_{5} \mathrm{NO}\right] \cdot 2 \mathrm{H}_{2} \mathrm{O}(0.0022)$ \\
(pro Probe, in g) & $\mathrm{K}_{2} \mathrm{CO}_{3}(0.013)$ \\
& $\mathrm{Na}_{2} \mathrm{HPO}_{4} \cdot 2 \mathrm{H}_{2} \mathrm{O}(0.02)$ \\
& $\mathrm{NaCl}(0.03)$ \\
& $\left.(\mathrm{Heweten} \mathrm{ad}(0.15))^{1}\right)$ \\
Probevolumen & $1 \mathrm{ml}$ Urin \\
Spezifität $\left.{ }^{2}\right)$ & nur für Cystein \\
Reaktion & kirschrote Farbe \\
Inkubationszeit & sofort \\
\hline
\end{tabular}

1) Kristalline Cellulose im Falle von Tablettenfertigung

$\left.{ }^{2}\right)$ Die Sulfhydrylpräparate Thiola ${ }^{\oplus}$ und $D$-Penicillamin (siehe Einführung) werden von der Methode nicht erfaßt (28)

Die semiquantitativen Bestimmungen der Konzentration werden gegen Bezugstafeln vorgenommen, die unter Zugrundelegung entsprechender Farbreaktionen in Urinvergleichslösungen bekannter Konzentrationsabstufungen von 0,$17 ; 0,41 ; 0,83$; 1,$24 ; 1,65 ; 2,07$ und $2,48 \mathrm{mmol}$ Cystein/1 $\left.(20-300 \mathrm{mg} / \mathrm{l})^{1}\right)$ angefertigt wurden.

1) Die ungewöhnlichen Abstufungen der Millimol-Konzentrationswerte von Cystein ergeben sich aus den primär gewählten Milligramm-Einwaagen. Dies gilt auch für die Angaben in den Tabellen 2, 3 und 4 (Umrechnungsfaktor $\mathrm{mg}$ in $\mathrm{mmol}$ 0,00826; $1 \mathrm{mmol}$ Cystein = $121 \mathrm{mg}$ ).

\section{Statistische Auswertung}

Präzision: In cystinarmen Urinen $(\leqslant 0,083 \mathrm{mmol} / \mathrm{l})$ werden Cysteinmengen nachfolgender Konzentrationsreihe von 0; 0,25; 0,$41 ; 0,66 ; 0,83 ; 0,99 ; 1,24 ; 1,65 ; 2,07$ und $2,48 \mathrm{mmol} / \mathrm{l}(0 ; 30$; $50 ; 80 ; 100 ; 120 ; 150 ; 200 ; 250$ und $300 \mathrm{mg} / 1)^{1}$ ) gelöst. Die semiquantitative Bestimmung mit dem Schnelltest entsprechend "Durchführung" wurde für jede Probe innerhalb 2 Stunden $12 \mathrm{mal}$ wiederholt, aus den Einzelwerten wurden $\bar{x} \pm s$ sowie VK\% bestimmt (Tab. 2).

Wiederfindung: Von 74 frischen Urinen mit bekannten Cysteineinwaagen (Soll) wurden die Wiederfindungskontrollen mit der unter "Durchführung" angeführten $\mathrm{K}_{2} \mathrm{CO}_{3} /$ Nitroprussid-Reagenzmischung in Pulverform (s. Tab. 1) vorgenommen. Die Sollwerte wurden in 4 Konzentrationsbereiche $(0,83-\mathrm{mmol}(100-$ mg)-Abstufungen) eingeteilt und die nach dem Schnelltest ermittelten Einzelwerte den Werten der Einzeleinwaagen vergleichend gegenübergestellt. In Abbildung 1 wird der Vergleich anhand der Mittelwerte $\bar{x}$ Cystein (Soll) und $\bar{x}$ Cystein (gefunden) geführt.

\section{Stabilitätsuntersuchungen}

Cysteinbezugsurine wurden jeweils bei Temperaturen von $25^{\circ} \mathrm{C}$ und $4{ }^{\circ} \mathrm{C}$ gelagert und Cysteinbestimmungen nach 24 Stunden und 3 Tagen gemäß „Durchführung“ vorgenommen. Die Stabilitätsbetrachtungen erfolgten im Vergleich zum Ausgangswert der frischen Lösungen (Tab. 3). Ebenso wurde mit einer quantitativen kolorimetrischen Cystinbestimmungsmethode die falschpsoitive Cystinanzeige von Cystein in Abhängigkeit von der Lagerungstemperatur und -zeit verfolgt.

Cystinbestimmung (Quantitative kolorimetrische Methode (27))

\section{Reaktionsprinzip und Durchführung}

Die Bestimmung entspricht im Reaktionsprinzip der „Brand'schen Probe“, wobei Cystin zunächst gemäß Gleichung 2 durch Cyanidionen gespalten wird.

$$
\mathrm{R}-\mathrm{S}-\mathrm{S}-\mathrm{R}+\mathrm{CN}^{-} \rightarrow \mathrm{R}-\mathrm{S}^{-}+\mathrm{RSCN}
$$

Die anschließende Farbreaktion des gebildeten Cysteins erfolgt entsprechend Gleichung 1.

$10 \mathrm{ml}$ Urin werden mit $100 \mathrm{mg}$ festem Ätznatron $(\mathrm{NaOH})$ bis $\mathrm{zu}$ dessen völliger Auflösung gerührt. Nach $15 \mathrm{~min}$ wird von ausgeflocktem Phosphat abfiltriert. Von dieser so präparierten Urinprobe werden $500 \mu \mathrm{l} \mathrm{mit} 2 \mathrm{ml} 0,05 \mathrm{~mol} / 1$ Triethylaminlösung versetzt und gut durchmischt. Anschließend werden gleichzeitig $500 \mu \mathrm{l}$ Natriumcyanidlösung $(50 \mathrm{~g} / \mathrm{l})$ und $1000 \mu \mathrm{l}$ frisch bereitete Nitroprussidlösung $(50 \mathrm{~g} / \mathrm{l})$ zugegeben. Es wird erneut gut durchmischt und die Absorption im Photometer nach genau 16 min Inkubationszeit gegen Wasser als Leerwert gemessen $(\mathrm{d}=1,0 \mathrm{~cm}, \lambda=535 \mathrm{~nm}$ ). Die Bestimmung der Konzentration erfolgt gegen eine aus Vergleichsurinen ermittelte Beżugskurve.

\section{Ergebnisse}

In Tabelle 2 sind als Maß für die Qualität der überprüften Schnelltestmethode die errechneten Mittelwerte, Standardabweichungen und Variationskoeffizienten für 10 Urine variierter Cysteinkonżentrationen zusammengestellt. Die Variationskoeffizienten liegen in den unteren Konzentrationsbereichen bis 1,65 $\mathrm{mmol} / \mathrm{l}$ relativ hoch (VK $=23-35 \%$ ). Mit zunehmender Cysteinkonzentration wird die Reproduzier- 
Tab. 2. Untersuchungen zur Präzision und Reproduzierbarkeit des Cystein-Schnelltestes an Urinen mit abgestuften Cysteinkonzentrationen (12 Wiederholungsmessungen) (Einwaage in $\mathrm{mg} / \mathrm{l}$ von $0-300$, Umrechnungsfaktor in $\mathrm{mmol} / \mathrm{l} 0,00826$ )

\begin{tabular}{llll}
\hline \multicolumn{3}{l}{ Cystcinkonzentration (mmol/l) } \\
\hline Einwaagc & \multicolumn{2}{l}{ gefunden } & VK \% \\
\cline { 2 - 3 } & $\overline{\mathbf{x}}$ & $\pm \mathrm{s}$ & \\
\hline 0 & 0 & 0 & 0 \\
0,25 & 0,35 & 0,10 & 29 \\
0,41 & 0,48 & 0,17 & 35 \\
0,66 & 0,69 & 0,21 & 30 \\
0,83 & 0,79 & 0,21 & 27 \\
0,99 & 0,93 & 0,27 & 29 \\
1,24 & 1,17 & 0,31 & 26 \\
1,65 & 1,55 & 0,35 & 23 \\
2,07 & 1,97 & 0,41 & 21 \\
2,48 & 2,27 & 0,38 & 17 \\
\hline
\end{tabular}

barkeit der Werte sichtlich besser. Der Nachweis von $0,083 \mathrm{mmol}(10 \mathrm{mg})$ in vitro gelöstem Cystein pro Liter Urin kann bereits an einer sichtbaren Rosafärbung der Reagenz/Probe-Mischung gesichert werden. In Abbildung 1 wird ein Vergleich von Cysteinwerten, die mit der eingesetzten $\mathrm{K}_{2} \mathrm{CO}_{3} /$ Nitroprossud-Reagenzmischung ermittelt wurden, gegenüber vorgegebenen Cysteineinwaagen vorgenommen (siehe „Sta-

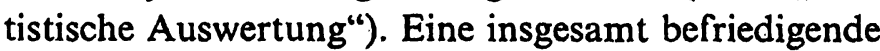
Übereinstimmung der Mittelwerte in den 4 Konzentrationsbereichen ist erkennbar, trotz z.T. größerer Einzelwertdifferenzen. So wurden mit dem Pulverreagenz durchschnittlich $82,2 \%$ der Cysteinsollwerte wiedergefunden. Die relativ hohen mittleren Abweichungen $(0,41-0,58 \mathrm{mmol} / \mathrm{l})$ in den oberen Konzentrationsbereichen sind in der zunehmenden Farbdichte der Reaktionslösungen begründet. Die Einzelabweichungen belaufen sich hierbei auf maximal $-0,83$ $\mathrm{mmol} / \mathrm{l} \mathrm{bzw}$. $+0,41 \mathrm{mmol} / \mathrm{l}$.

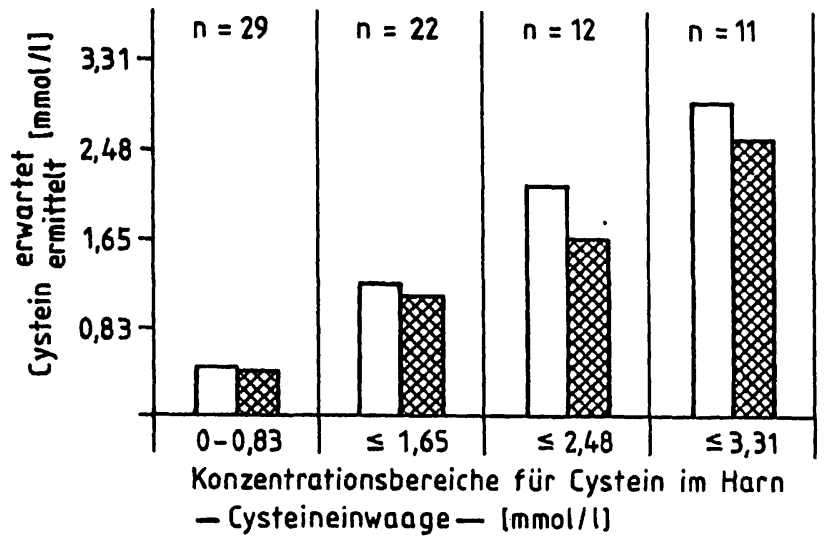

Abb. 1. Mit dem $\mathrm{K}_{2} \mathrm{CO}_{3} /$ Nitroprussid-Test geführte Wiederfindungen von Cysteineinwaagen (Sollwerte) in Urinen. Gegenüberstellung der Mittelwerte $\bar{x}$ Cystein (Soll) und $\overline{\mathbf{x}}$ Cystein (ermittelt) über 4 Konzentrationsberciche.

\section{$\square \quad$ Einwaage \\ Testergebnis}

In Tabelle 3 ist das Verhalten von anfänglich frischen Cystein enthaltenden Vergleichsurinen in Abhängigkeit von der Lagerungszeit und -temperatur dargestellt. Erwartungsgemä $B$ ist die Sofortreaktion nach Zugabe der $\mathrm{K}_{2} \mathrm{CO}_{3} / \mathrm{Nitroprussid-Testmischung} \mathrm{sehr}$ deutlich und entspricht in ihrer Intensität der vorgelegten Cysteinkonzentration. Schon nach 1 Tag Lagerungszeit bei $25^{\circ} \mathrm{C}$ werden die Farbintensitäten besonders im Konzentrationsbereich bis $1,65 \mathrm{mmol} / \mathrm{l}$ $(200 \mathrm{mg} / \mathrm{l})$ - deutlich geringer. Nach 3 Tagen ist ein Cysteinnachweis nicht mehr oder lediglich in Spuren zu führen. Eine Lagerungstemperatur von $4{ }^{\circ} \mathrm{C}$ ändert an diesem Verhalten nur wenig. In früheren Studien konnten wir eine während der Lagerung erfolgte nahezu vollständige Oxidation von Cystein zur Disulfidverbindung Cystin veranschaulichen (28), vgl. auch Fußnote a) in Tabelle 3.

Tab. 3. Stabilität von Cystein im Urin in Abhängigkeit von verschicdenen Lagcrungsbedingungen (Temperatur, Zeit) sowic Einfluß von Cysteinzusätzen auf Cystinbestimmungen (mmol/l)

\begin{tabular}{|c|c|c|c|c|c|c|c|c|c|}
\hline \multirow{3}{*}{$\begin{array}{l}\text { Cystein- } \\
\text { Vergleichslösung } \\
\text { mmol/1 } \\
\text {. }\end{array}$} & \multicolumn{2}{|l|}{$\mathrm{t}=0$} & \multicolumn{2}{|c|}{$\begin{array}{l}\mathrm{t}=1 \mathrm{Tag} \\
25^{\circ} \mathrm{C}\end{array}$} & \multirow[t]{2}{*}{$4^{\circ} \mathrm{C}$} & \multicolumn{3}{|c|}{$\begin{array}{l}\mathrm{t}=3 \text { Tage } \\
25^{\circ} \mathrm{C}\end{array}$} & \multirow[t]{2}{*}{$4^{\circ} \mathrm{C}$} \\
\hline & \multicolumn{2}{|l|}{ Methode } & \multicolumn{2}{|l|}{ Methode } & & \multicolumn{3}{|c|}{ Methode } & \\
\hline & 1 & 2 & 1 & 2 & 2 & $\overline{1}$ & $\left.2^{a}\right)$ & 1 & 2 \\
\hline $\begin{array}{l}0 \\
0,41 \\
0,83 \\
1,24 \\
1,65 \\
2,07 \\
2,48\end{array}$ & $\begin{array}{l}- \\
+ \\
++ \\
++t \\
+++t \\
+++t \\
+++t\end{array}$ & $\begin{array}{l}0 \\
0,48 \\
0,88 \\
1,48 \\
2,08 \\
2,88 \\
3,13\end{array}$ & $\begin{array}{l}- \\
(+) \\
+ \\
++ \\
+++ \\
++++ \\
++++\end{array}$ & $\begin{array}{l}0 \\
0,28 \\
0,48 \\
0,79 \\
1,29 \\
1,60 \\
1,96\end{array}$ & $\begin{array}{l}0 \\
0,34 \\
0,51 \\
0,88 \\
1,31 \\
1,73 \\
2,55\end{array}$ & $\begin{array}{l}- \\
- \\
- \\
\overline{(+)} \\
(+)\end{array}$ & $\begin{array}{l}0 \\
0,25 \\
0,44 \\
0,70 \\
0,81 \\
1,04 \\
1,38\end{array}$ & $\begin{array}{l}- \\
- \\
- \\
(+) \\
(+) \\
+ \\
++\end{array}$ & $\begin{array}{l}0 \\
0,32 \\
0,52 \\
0,69 \\
0,95 \\
1,28 \\
1,53\end{array}$ \\
\hline
\end{tabular}

Methode 1: siehe "Cystein-Schnelltest" mit $\mathrm{K}_{2} \mathrm{CO}_{3} /$ Nitroprussid

2: siehe "Cystinbestimmung" mit $\mathrm{CN}^{-} /$Nitroprussid gegen Cystin-Bezugskurve (Cystinwertanzeige!)

") nahezu vollständige Oxidation von Cystein zu Cystin 
Ein anderer interessanter Aspekt findet sich in Reaktionen der frischen Cystein enthaltenden Vergleichsurine mit den Reagenzien zur quantitativen Cystinbestimmung (siehe "Cystinbestimmung“). Diese falschpositiven Cystinreaktionen fallen im getesteten Cysteinkonzentrationsbereich von $0,41-2,48 \mathrm{mmol} / \mathrm{l}$ $(50-300 \mathrm{mg} / \mathrm{l})$ sehr stark aus (Tab. $3 ; \mathrm{t}=0)$ und entsprechen ungefähr dem aus der vorgelegten $\mathrm{Cy}$ steinmenge rückläufig ableitbaren Moläquivalent an Cystin. Eine Erklärung hierfür ist in den Reaktionsmechanismen der Cystinbestimmungsmethode zu finden, nach denen Cystin durch Cyanidionen äquimolar in Cystein überführt und dieses durch Nitroprussid kolorimetrisch angezeigt wird (vgl. in der Reihenfolge Gleichungen 2 und 1). Aus der jeweiligen Farbintensität der Reaktionslösung wird dann über die Cystinkonzentrations-Bezugskurve auf den entsprechenden Cystinwert geschlossen (siehe "Cystinbestimmung").

Die beschriebenen analytischen Befunde haben uns nun zu der Überlegung geführt, durch Kombination der beiden verwendeten Bestimmungsmethoden eine semiquantitative Differenzierung von Cystin und $\mathrm{Cy}-$ stein nebeneinander zu führen. In Tabelle 4 wird ein praktisches Beispiel dieser getrennten Gehaltsbestimmung in Urinen demonstriert. Einem Vergleichsurin mit $1,25 \mathrm{mmol}$ Cystin pro Liter $(300 \mathrm{mg} / \mathrm{l})$ wurde Cystein in Konzentrationen von je 0,41; 0,83 und 1,24 $\mathrm{mmol} / \mathrm{l}(50 ; 100 ; 150 \mathrm{mg} / \mathrm{l})$ zugemischt. In der Mischung addiert sich der durch Cystein jeweils bedingte Anteil an falschpositiven Cystinwerten der vorgegebenen Cystinkonzentration von $1,25 \mathrm{mmol} / \mathrm{l} \mathrm{zu}$ und entspricht nach der quantitativen Cystin-Bestimmungsmethode mit $\mathrm{CN}^{-} /$Nitroprussid dem Moläquivalent an vorgelegtem Cystein. Mit dem Pulverrea- genz $\mathrm{K}_{2} \mathrm{CO}_{3} / \mathrm{Nitroprussid} \mathrm{werden} \mathrm{die} \mathrm{tatsächlichen}$ molaren Konzentrationen an Cystein aus der CystinCystein-Mischung ermittelt. Nach Abzug dieser Werte von den falschpositiven Cystinwerten ergibt sich annähernd die vorgegebene Cystinkonzentration von $1,25 \mathrm{mmol} / \mathrm{l}$. Die mittlere Cystinwiederfindung beläuft sich im vorgestellten Beispiel auf $108 \%$.

\section{Diskussion}

Die Schnelltestmethode mit der $\mathrm{K}_{2} \mathrm{CO}_{3} /$ NitroprussidReagenzmischung gestattet eine semiquantitative Schnellinformation zur Cysteinkonzentration im Urin. Die Werte für VK in Tabelle 2 machen insbesondere in den unteren Konzentrationsbereichen bis $1,65 \mathrm{mmol} / 1(200 \mathrm{mg} / \mathrm{l})$ eine relativ hohe Ungenauigkeit deutlich, was sicherlich in den $0,41 \mathrm{mmol}(50$ $\mathrm{mg}$ )-Abstufungen der Farbbezugstafeln begründet ist. Dies sowie die in Abbildung 1 dargestellten Abweichungen der Wiederfindungsraten von den Sollwerten der Einwaagen sollten aber für die Erfordernisse einer laboratoriumsdiagnostischen Verlaufskontrolle bei Ascorbinsäuretherapie weniger von Gewicht sein, wenn wir in der Lage sind, Cysteinkonzentrationen zwischen negativ bis mäßig erhöht $=0-0,41 \mathrm{mmol} / 1$ $(0-50 \mathrm{mg} / \mathrm{l})$; mittelstark $=0,41-1,24 \mathrm{mmol} / 1(150$ $\mathrm{mg} / \mathrm{l})$; stark $=1,24-2,48 \mathrm{mmol} / \mathrm{l}(300 \mathrm{mg} / \mathrm{l})$ und sehr stark $>2,48 \mathrm{mmol} / 1$ zuverlässig zu unterscheiden. Eine Nachweisgrenze von 0,083-0,12 mmol Cystein pro Liter Urin $(10-15 \mathrm{mg})$ ist gesichert. Für Normalurin sind Cysteinwerte von $0,0025-0,050 \mathrm{mmol} / 1$ $(0,3-6 \mathrm{mg} / \mathrm{l})$ beschrieben $(4,29,30)$. Mit der von uns eingesetzten Cystein-Schnelltestmethode konnten wir zunächst in Normalurinen - und selbst in Urinen

Tab. 4. Beispiel der getrennten semiquantitativen Ermittlung von Cystin und Cystein im Urin (siehe "Cystein-Schnelltest“" und "Cystinbestimmung") - Angaben in $\mathrm{mmol} / \mathrm{l}(\mathrm{mg} / \mathrm{l})$

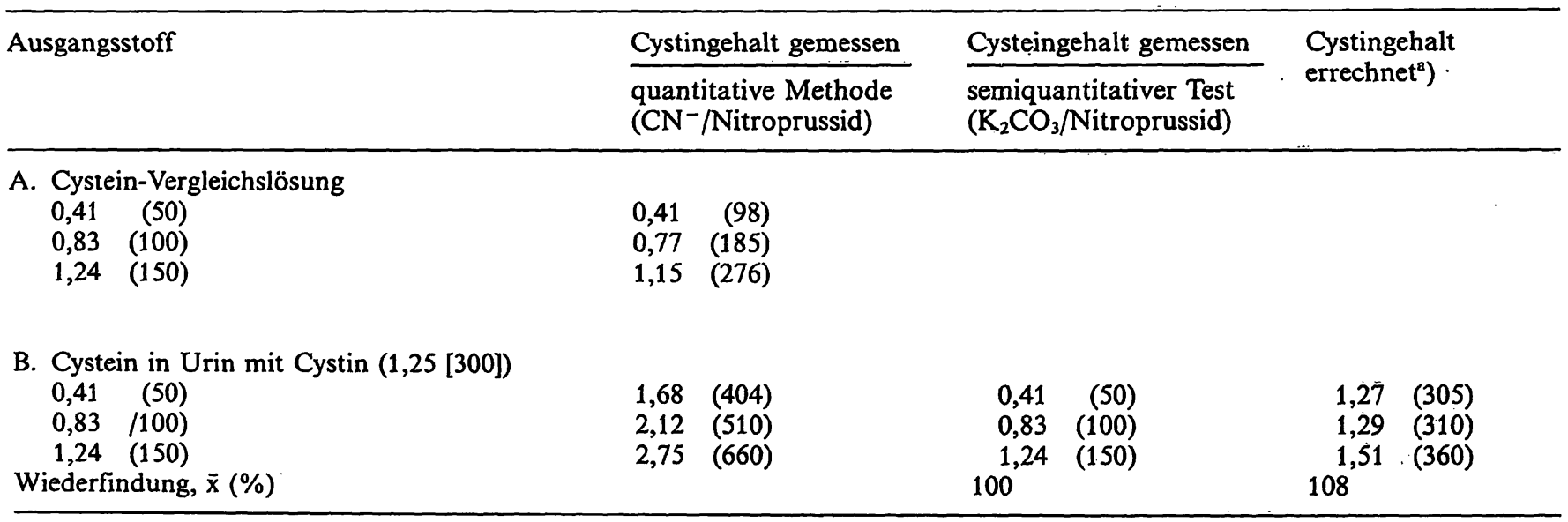

Aussagen:

A. Falschpositive Cystinanzeige durch Cysteineinwaage; 1 mol Cystein $(121 \mathrm{~g}) \cong \sim 1 \mathrm{~mol}$ Cystin $(240 \mathrm{~g})$.

B. Eine getrennte semiquantitative Bestimmung von Cystin und Cystein aus Cystin/Cysteinmischungen (frische .Urine!) ist möglich a) Cystin gemessen ( $\mathrm{mmol} / \mathrm{l})$ abzüglich Cystein gemessen $(\mathrm{mmol} / \mathrm{l})$ 
von Cystinurikern ohne medikamentöse Behandlung - keinen Cysteinnachweis führen. Dies wiederum unterstreicht, daß ein positiver Cysteinbefund mit dem $\mathrm{K}_{2} \mathrm{CO}_{3} /$ Nitroprussid-Test allein schon eine deutliche Cysteinerhöhung im Urin signalisiert. In ersten orientierenden Untersuchungen bei Cystinurikern unter Ascorbinsäuretherapie konnten wir Cysteinkonzentrationswerte von 0,41 bis $1,65 \mathrm{mmol} / 1$ ermitteln (28).

Zur Cysteinbestimmung sollten stets frische Urine kommen, da geringe Cysteinmengen schon innerhalb kurzer Lagerungszeiten von 24 Stunden durch Oxidation mit Luftsauerstoff beträchtliche Umwandlung zu Cystin erfahren können $(4,29)$ (vgl. auch Tab. 3). Deshalb ist eine Untersuchung von Portionsurinen angeraten. In Ausnahmefällen empfehlen wir Kühlschranklagerung der Urine bei $4^{\circ} \mathrm{C}$ für max. 1 Tag.

Cystein ergibt mit der quantitativen Cysteinbestimmungsmethode (Cyanid/Nitroprussid) stark positive Cystinbefunde. Ein quasianaloges Reaktionsverhalten

\section{Literatur}

1. Křižek, V. (1976) Zschr. Urol. Nephrol. 69, 369-374.

2. Matouschek, E. \& Hubert, R. D. (1981) Urolithiasis Pathogenese, Diagnostik und Therapie; F. K. Schattauer Verlag Stuttgart-New York, S. 142-151.

3. Rosenberg, L. E., Downing, S. U., Durant, J. \& Segal, S. (1966) J. Clin. Invest. 45, 365-371.

4. Asper, R. \& Schmucki, O. (1982) Urol. Int. 37, 91-109.

5. Berg, Ch. (1983) Auswertung von 100000 Harnsteinanalysen in der DDR, Dissertation, Friedrich-Schiller-Universität Jena.

6. Kallistratos, G. \& Malorny, G. (1972) Arzneim.-Forsch. $22,1434-1444$.

7. Lux, B. \& May, P. (1980) Zystinstein; In: Urolithiasis 5 (Vahlensieck, W., ed.) Springer-Verlag Berlin-Heidelberg-New York, S. 40-52.

8. Mulvaney, W. P., Quilter, T. \& Mortera, A. (1975) J. Urol. $114107-108$.

9. Werner, F. \& Weinmann, H. M. (1966) Med. Klinik 61, $1827-1831$.

10. Hautmann, R. (1986) Zystinurie; Spezielle Maßnahmen beim Zystinstein. In: Harnsteinfibel (Hautmann, R. \& Lutzeyer, W., eds.) Deutscher Ärzteverlag Köln, S. 114-118 u. $208-217$.

11. Knudsen, L. \& Weismann, K. (1978) Acta Med. Scand. 204, 75-79.

12. Miano, L., Gallucci, M. \& Petti, S. (1979) Eur. Urol. 5 $265-272$.

13. Vivien, P., Allanic H. Turpin, J. Prunier, P., Laborgne, P. \& Loussouern, J. (1971) Therapie 26, 121-127.

14. Asper, R., Eggli, R. \& Schmucki, O. (1978) Fortschr. Urol. Nephrol. 11, 178 - 182 . von Cystein konnten wir in anderen Studien auch mit dem „Urocystin“ $\left(\mathrm{Ni}^{2+} / \mathrm{S}_{2} \mathrm{O}_{4}{ }^{2-}\right)$-Schnelltest (31) beobachten (28). Mit den beiden eingesetzten Methoden $\mathrm{CN}^{-} /$Nitroprussid für Cystin und $\mathrm{K}_{2} \mathrm{CO}_{3} /$ Nitroprussid für Cystein ist eine getrennte semiquantitative Cystin- und Cysteinbestimmung in Urinen möglich (siehe Tab. 4). Auch hierbei ist auf die Verwendung frischer Urine zu achten, da Cystinzersetzungsprodukte $\left(\mathrm{H}_{2} \mathrm{~S}\right)$ mit dem Cystein-Schnelltest sofort $\mathrm{zu}$ artefiziellen Farbreaktionen führen (28)!

Aus den vorliegenden Untersuchungsergebnissen ist eine einfache Anwendung des beschriebenen methodischen Vorgehens für gezielte Verlaufskontrollen der Cystein- und Cystinprofile in Urinen von Cystinurikern bei therapeutischer Anwendung von Ascorbinsäure ableitbar. Daraus könnten sich auch gegenüber den bisher üblichen Langzeitbeobachtungen zur Rezidivhäufigkeit von Cystinharnsteinen $(4,15,16)$ frühzeitig metaphylaktische Konsequenzen ergeben.

15. Brundig, P., Börner, R.-H., Berg, W., Pirlich, W., Böhm, W. D., Hoffmann, L. \& Klein, B. (1986) Zschr. Urol. Nephrol. 11, 178-182.

16. Lux, P. \& May, P. (1983) Urol. Int. 38, 91-94.

17. Pendse, A. K., Purohit, A. K., Grosh, R., Goyal, A. \& Singh, P. P. (1984) Urol. Res. 12, 62-65.

18. Schmucki, O. \& Asper, R. 81982) Akt. Urol. 13, $81-83$.

19. Eggli, R. \& Asper, R. (1978) Anal. Chim. Acta 101, 253259.

20. Haux, P. \& Natelson, S. (1970) Clin. Chem. 16, 366-369.

21. Kolthoff, J. M. \& Kihara, S. (1977) Analyt. Chem. 49, 2108-2109.

22. Saetre, R. \& Rabenstein, D. L. (1978) Analyt. Biochem. 90, 684-692.

23. Roesel, R. A. \& Coryell, M. E. (1974) Clin. Chim. Acta $52,343-346$.

24. Sullivan, M. X., Hess, W. C. \& Howard, H. W. (1942) J. Biol. Chem. 145, 621-624.

25. Brand, E., Harris, M. M. \& Biloon, S. (1930) J. Biol. Chem. $86,315-331$.

26. Maaser, M., Hesse, A., Linke, E., Klare, S. \& Schneider, H.-J. (1972) Dtsch. Ges.-Wesen 27, 1119-1121.

27. Berg, W. \& Hesse, A. (1975) Z. Med. Labortechn. 16, 315319.

28. Kilian, O. (1985) Schnell- und Suchtest auf Cystin und Cystein im Urin; Diplomarbeil, Friedrich-Schiller-Universität Jena.

29. Asper, R., Schmucki, O., Eggli, R. \& Rosenmund, H. (1979) Fortschr. Urol. Nephrol. 14, 417-421.

30. Brigham, P., Stein, W. H. \& Morre, S. (1960) J. Clin. Invest. 39, 1633-1637.

31. Kinoshita, K., Takemoto, M., Itatani, H.. Yachiku, S., Kotake, T. \& Sonoda, T. (1979) Urol. Int. 34, 369-373.
Dr. sc. nat. W. Berg

Klinik und Poliklinik für Urologie

Bereich Medizin der

Friedrich-Schiller-Universität Jena

Lessingstr. 1

DDR-6900 Jena 
